\title{
Evolução dos Níveis de Colesterol na População Adulta de São José do Rio Preto (1991-1997)
}

\author{
José Carlos Nicolau, Cristiani Nogueira, Lília Nigro Maia, José Antonio Franchini Ramires
}

\author{
São Paulo, SP - São José do Rio Preto - SP
}

Objetivo - Realizar levantamento de fatores de risco para aterosclerose na população $\geq 20$ anos de São José do Rio Preto, e comparar os dados obtidos com os coletados, em levantamento similar, em 1991.

Métodos - Pesquisa quantitativa com amostragem estratificada por sexo e idade. Os entrevistados (646 individuos, 303 homens) foram abordados em Postos de Saúde e outros pontos de afluxo da população, distribuídos por zona geográfica e por classe social dos bairros. A margem de erro foi de 4\%, e o intervalo de confiança (IC) de $95 \%$.

Resultados - A) Níveis médios de HDL-colesterol (apenas em 1997): homens 43,7 $\pm 15 \mathrm{mg} / \mathrm{dL}$, mulheres 49,6 $\pm 13,5 \mathrm{mg} / \mathrm{dL}(\mathrm{p}<0,001,95 \%$ IC 3,7 a 8,1); B) niveis médios de colesterol total em 1991 vs 1997: população global 192,5 $\pm 48,9 \mathrm{mg} / \mathrm{dL}$ vs $190,5 \pm 42,5 \mathrm{mg} / \mathrm{dL}(p=N S)$; homens 187,6 $\pm 53,3$ vs 190,5 $\pm 42,5 \mathrm{mg} / \mathrm{dL}(p=N S)$; mulheres $196,8 \pm 40 \mathrm{mg} / \mathrm{dL}$ vs $187,6 \pm 37,8 \mathrm{mg} / \mathrm{dL}(p=0,008,95 \%$ IC 2,4 a 15,9). C) Em análise de regressão, as variáveis que se correlacionaram significativamente com níveis de colesterol, em ambas as pesquisas, foram: idade $(p<0,001)$, pressão arterial sistólica $(p<0,001) e$ diastólica $(p<0,001)$, dieta $(p<0,001)$. Sexo feminino correlacionou-se apenas em $1991(p=0,011)$ e sedentarismo apenas em 1997 ( $p=0,014)$.

Conclusão - Os níveis de colesterol na população adulta de São José do Rio Preto são bastante aceitáveis, sendo que no sexo feminino diminuíram, de maneira significativa, na pesquisa atual, em relação à realizada em 1991.

Palavras-chave: fatores de risco, colesterol, levantamento populacional

\section{Trends in Cholesterol Levels in the Adult Population of São José do Rio Preto (1991-1997)}

Purpose - To develop a survey about risk factors for atherosclerosis in $a \geq 20$-year-old population from São José do Rio Preto, and compare the results with those obtained in a similar survey in 1991.

Methods - Quantitative survey with sample stratified by sex and age. The individuals (a total of 646, 303 men) where contacted in outpatient facilities from the Health Secretary and other populated sites, distributed by geographic zone and social class of different neighborhoods. The standard error of the survey was 4\%, and the confidence interval was $95 \%$.

Results - A) Mean HDL-cholesterol (only in 1997): male gender $43.7 \pm 15 \mathrm{mg} / \mathrm{dL}$, female gender $49.6 \pm 13.5 \mathrm{mg} / \mathrm{dL}$ $(p<0.001,95 \%$ CI 3.7 a 8.1). B) Mean total cholesterol in 1991 vs 1997: for the global population 192.5 $\pm 48.9 \mathrm{mg} / \mathrm{dL}$ vs $190.5 \pm 42.5 \mathrm{mg} / \mathrm{dL}(\mathrm{p}=\mathrm{NS})$; for men $187.6 \pm 53.3 \mathrm{mg} / \mathrm{dL}$ vs $190.5 \pm 42.5 \mathrm{mg} / \mathrm{dL}(\mathrm{p}=\mathrm{NS}) ;$ for women $196.8 \pm 40 \mathrm{mg} / \mathrm{dL} v \mathrm{vs}$ $187.6 \pm 37.8 \mathrm{mg} / \mathrm{dL}(p=0.008,95 \%$ CI 2.4 a 15.9). C) By regression analysis, the variables that correlated significantly with cholesterol levels, in both surveys, were: age $(p<0.001)$, systolic $(p<0.001)$ and diastolic $(p<0.001)$ arterial pressure, diet $(p<0.001)$. Female gender showed correlation only in the 1991 survey $(p=0.011)$, and sedentarism only in $1997(p=0.014)$.

Conclusion - The mean cholesterol levels in the adult population of São José do Rio Preto are very favorable and, in female gender, showed a significant decrease in the 1997 survey, relatively to the 1991 survey.

Key-words: risk factors, cholesterol, population survey

Arq Bras Cardiol, volume 71 (no 5), 699-704, 1998

Instituto do Coração do Hospital das Clínicas-FMUSP, São Paulo - Hospital de Base da Faculdade de Medicina de São José do Rio Preto-FAMERP e Instituto de Moléstias Cardiovasculares-IMC - São José do Rio Preto Correspondência: José Carlos Nicolau - Rua Aureliano Coutinho, 355 - $14^{\circ}$ 01224-020 - São Paulo, SP

Recebido para publicação em 29/6/98

Aceito em 9/9/98
Segundo dados da Organização Mundial da Saúde ${ }^{1,2}$, as doenças cardiovasculares (DCV) são responsáveis por 12 milhões de óbitos, anualmente, em todo o mundo, causando, aproximadamente, metade dos óbitos em diversos países desenvolvidos, sendo a principal causa de óbito em vários países em desenvolvimento e a maior causa de óbito 
na população adulta. Além disso, sabe-se que as DCVs podem afetar, importantemente, a habilidade funcional, apesar deste dado ser de difícil mensuração. Nas Américas, estimase em 800 mil o número de óbitos/ano, com aproximadamente a mesma proporção de homens e mulheres. Por outro lado, vem se demonstrando, nas últimas décadas, uma diminuição na mortalidade por DCV, de maneira geral, apesar desse declínio não ser uniforme. Por exemplo, os Estados Unidos da América (EUA), Austrália, Canadá e França conseguiram declínio na mortalidade da ordem de $50 \%$, ao passo que em países como Polônia e Bulgária, a incidência aumentou em mais de 50\%. Importante notar que nos EUA, a metade dos óbitos cardiovasculares são devidos à doença isquêmica do coração (DIC) ${ }^{3}$.

No Brasil ${ }^{4}$, as DCVs são responsáveis por $34 \%$ dos óbitos globais, sendo aproximadamente $1 / 3$ por DIC. Em relação à evolução temporal destes índices em nosso meio ${ }^{5,6}$, os resultados não são uniformes. Especificamente no Estado de São Paulo, ao noroeste do qual se situa São José Rio Preto, está ocorrendo uma diminuição na mortalidade por DIC da ordem de $1,16 \%$ ao ano para homens, e de $1,52 \%$ ao ano para mulheres.

Por outro lado, desde há muito se sabe que o perfil lipídico, fundamentalmente o colesterol e suas frações, guarda relação direta com a DIC, demonstrando-se riscos cada vez mais elevados, quanto maior a colesterolemia, principalmente $>200 \mathrm{mg} / \mathrm{dL}$, o mesmo ocorrendo com a mortalidade ${ }^{7,8}$.

Em anos recentes, vem sendo demonstrado que o controle da dislipidemia é eficaz no sentido de se diminuir eventos, inclusive óbitos, tanto na prevenção primária quanto na secundária $^{9-11}$.

Apesar da importância do colesterol como fator de risco, em nosso país os estudos vêm focando grupos específicos de pacientes, como por exemplo, indivíduos trabalhadores em saúde, servidores públicos, advogados, metalúrgicos, médicos ${ }^{12-16}$, muito pouco existindo em termos populacionais, com ausência de dados sobre a evolução do perfil lipídico ao longo do tempo.

O intuito deste estudo foi o de realizar, pela primeira vez em nosso meio, uma comparação entre o perfil atual da população adulta de São José do Rio Preto, cidade de porte médio localizada em uma das regiões mais ricas do país, e o perfil obtido em levantamento similar realizado há seis anos atrás.

\section{Métodos}

Em população da cidade de São José do Rio Preto (SP) $\geq 20$ anos, foram selecionados 646 indivíduos, assim distribuídos: 303 homens e 343 mulheres, sendo 187 com idades entre 20 e 29 anos; 166 entre 30 e 39 anos; 115 entre 40 e 49 anos; 93 entre 50 e 59 anos e $85 \geq 60$ anos.

A metodologia da coleta de dados consta de comunicação descrita anteriormente ${ }^{17}$. Em resumo, durante a Semana do Coração de 1997, realizou-se pesquisa do tipo quantitativa, com amostragem estratificada por sexo e idade da população residente na cidade de São José do Rio Preto. Os entrevistados foram abordados em Postos de Saúde (todos participantes da Semana do Coração) e outros pontos de grande afluxo da população, distribuídos por zona geográfica e por classe social dos bairros.

A abordagem dos indivíduos foi realizada por pessoal especialmente contratado e treinado para a pesquisa que, além da coleta de sangue para dosagem de colesterol total e HDL-colesterol (HDL-c), e da medição da pressão arterial, aplicaram questionários sob treinamento e orientação de firma especializada (Datafolha). Especificamente em relação ao dado de estresse, levou-se em consideração a auto-avaliação do próprio indivíduo, que considerava seu estilo de vida nada, mais ou menos, ou muito estressante. Nas dosagens sangüíneas foram utilizados aparelhos portáteis Cholestech-LDX, obtendo-se a amostra sangüínea por punção digital. Saliente-se que, em pesquisa recente ${ }^{18}$ realizada em nosso meio, demonstrou-se que a precisão e exatidão obtidas com tal metodologia concordam com as recomendações do National Educational Program norte-americano, tanto para o colesterol total quanto para o HDL-c.

A margem de erro para esta pesquisa foi de $4 \%$, dentro de um intervalo de confiança (IC) de $95 \%$, significando que, se fossem feitos 100 levantamentos simultâneos com a mesma metodologia, em 95 casos os resultados estariam dentro da margem de erro prevista nesta pesquisa.

Os dados de estatística descritiva e as análises de regressão foram obtidos com o auxílio do programaStatistics, parte do SYSTAT ${ }^{19}$. Nas comparações entre médias utilizouse o teste t de Student para amostras não pareadas e, nas comparações entre proporções, o teste " $z$ " para comparações entre proporções de amostras. Estes dados foram obtidos com o auxílio do programa Primer of Biostatistics ${ }^{20}$.

Nos intervalos de confiança utilizou-se o limite de $95 \%$, e as diferenças foram consideradas significativas para $\mathrm{p} \leq 5 \%$ (bicaudal).

\section{Resultados}

As figuras 1 e 2 apresentam, respectivamente, os histogramas com a distribuição do colesterol total e HDL-c.

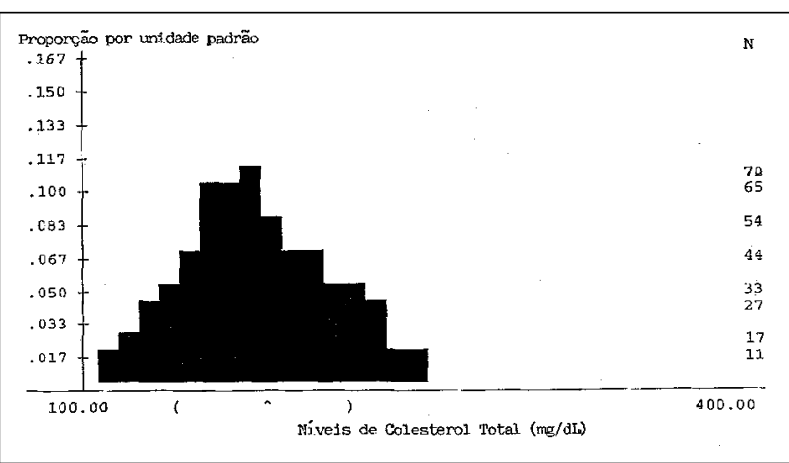

Fig. 1 - Distribuição dos níveis do colesterol total na população analisada* ^ $^{\wedge}=$ média ( ) desvio-padrão; proporção por unidade padrão=representa uma escala de densidade probabilística para distribuições com desvio-padrão $=1 ; \mathrm{N}=$ cada número corresponde à contagem máxima possível em qualquer barra, no mesmo nível; *dados de 1997. 


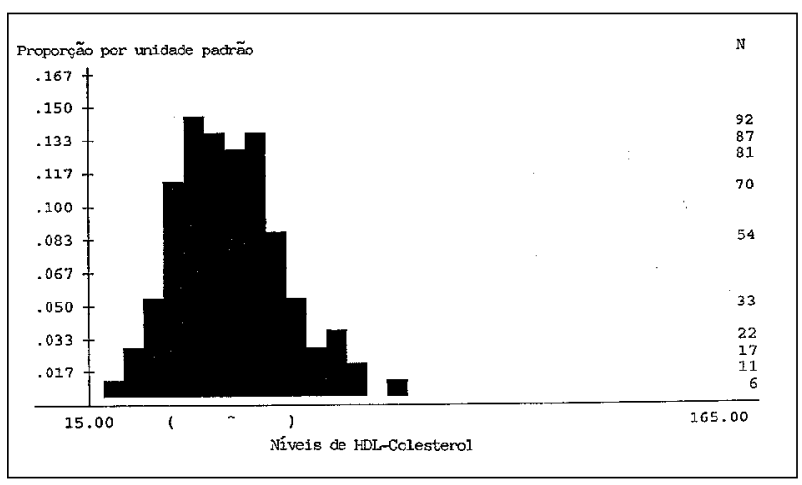

Fig. 2 - Distribuição dos níveis de HDL-colesterol na população analisada*. Ver explicações na figura $1 ; *$ dados de 1997.

A tabela I demonstra as características clínicas da população mais recentemente analisada. Duas diferenças importantes, em relação aos dados de 1991: a) na pesquisa anterior, o limite mínimo de idade foi de 30 anos, e na pesquisa atual, de 20 anos; b) na pesquisa anterior não foi dosado o HDL-c, ao contrário da atual.

A tabela II demonstra os níveis de colesterol total e de HDL-c na população, em relação a sexo e idade. Como se nota, o colesterol total médio nos homens foi de $180,3 \mathrm{mg} / \mathrm{dL}$, ao passo que nas mulheres foi de $179,9 \mathrm{mg} / \mathrm{dL}$ ( $\mathrm{p}=\mathrm{NS}$ ). Além disso, nota-se um aumento crescente nesses níveis até a $6^{\mathrm{a}}$ década nos homens, e em todas as faixas analisadas nas mulheres. Por outro lado, em relação ao HDL-c, seus níveis

\begin{tabular}{|lc|}
\hline \multicolumn{2}{|c|}{ Tabela I - Características clínicas da população analisada* } \\
\hline N & 646 \\
Idade média (anos) & $40,4 \pm 14,9$ \\
Colesterol total médio (mg/dL) & $180,1 \pm 41$ \\
HDL-colesterol médio (mg/dL) & $46,9 \pm 14,5$ \\
PAS média (mmHg) & $123,2 \pm 18,2$ \\
PAD média (mmHg) & $79,9 \pm 12$ \\
IMC médio (kg/altura ${ }^{2}$ & $25,7 \pm 6,7$ \\
Renda familiar média (R\$) & $1118,9 \pm 1330,6$ \\
Índice médio de escolaridade (1-8) & $3,3 \pm 2$ \\
Sexo masculino (\%) & 46,9 \\
Pacientes em dieta (\%) & 17,8 \\
Pacientes com vida sedentária (\%) & 66,2 \\
Pacientes fumantes (\%) & 24,3 \\
Pacientes sem estresse (\%) & 10,3 \\
\hline PAS- pressão arterial sistólica; PAD- pressão arterial diastólica; IMC- \\
índice de massa corporal; \pm desvio-padrão; *dados de 1997. \\
\hline
\end{tabular}

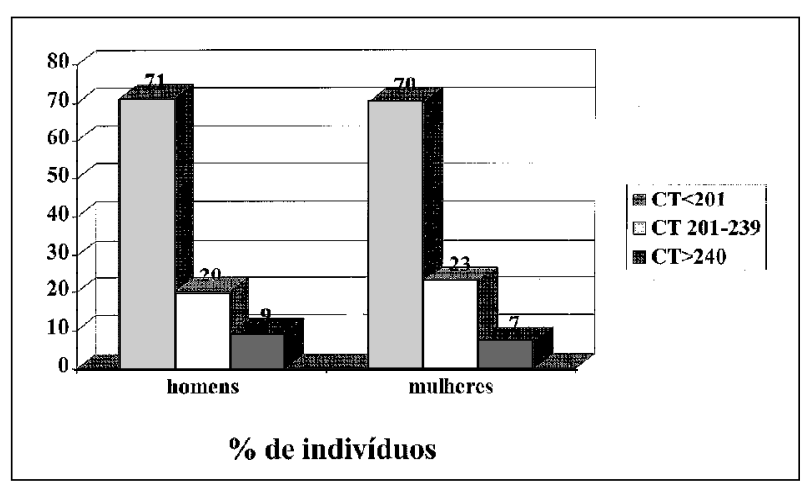

Fig. 3 - Prevalência de colesterol total desejável (<201mg/dL), intermediário (201$239 \mathrm{mg} / \mathrm{dL}$ ) e aumentado (>240mg/dL) na população analisada*; *dados de 1997.

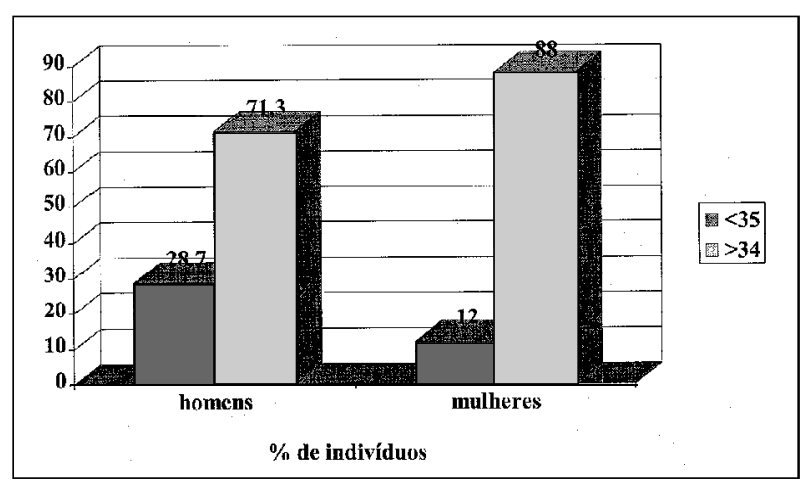

Fig. 4 - Prevalência de HDL-c ideal ( $\geq 35 \mathrm{mg} / \mathrm{dL})$ ou não $(<35 \mathrm{mg} / \mathrm{dL})$ na população analisada*; *dados de 1997.

médios são maiores nas mulheres, em relação aos homens, em quaisquer das faixas etárias analisadas. Entretanto, não foram encontradas diferenças significativas entre os níveis do HDL-c e as diferentes faixas etárias, tanto em homens, quanto em mulheres.

A figura 3 apresenta a prevalência de colesterol total desejável (<201mg/dL), intermediário (201-239mg/dL) e aumentado (>240mg/dL) nesta população. Da mesma forma, a figura $4 \mathrm{de}-$ monstra a prevalência de HDL-cideal $(\geq 35 \mathrm{mg} / \mathrm{dL})$ ou não.

A tabela III apresenta a evolução do perfil de colesterol entre 1991 e 1997. Como se nota, não houve alteração significativa entre os dois levantamentos em relação ao global da população, ou em relação ao sexo masculino. Entretanto, houve uma diminuição significativa nos níveis de colesterol total entre as mulheres $(\mathrm{p}=0,008)$.

\begin{tabular}{|c|c|c|c|c|c|c|}
\hline \multicolumn{7}{|c|}{ Tabela II - Níveis de colesterol total e HDL-colesterol em relação a sexo e idade } \\
\hline & \multicolumn{2}{|c|}{ Colesterol total $(\mathrm{mg} / \mathrm{dL})$} & \multirow[t]{2}{*}{$\mathrm{P}$} & \multicolumn{2}{|c|}{ HDL-colesterol (mg/dL) } & \multirow[t]{2}{*}{$\mathrm{P}$} \\
\hline & homens & mulheres & & homens & mulheres & \\
\hline 20-29 anos & $157 \pm 36,3$ & $160,2 \pm 34,1$ & 0,538 & $42,4 \pm 14,8$ & $51,3 \pm 13,6$ & $<0,001$ \\
\hline 30-39 anos & $181,1 \pm 39,9$ & $170 \pm 31,1$ & 0,048 & $45,6 \pm 16$ & $50,4 \pm 13,4$ & 0,037 \\
\hline $40-49$ anos & $200,3 \pm 45,2$ & $187,4 \pm 37,4$ & 0,098 & $44,2 \pm 17,1$ & $47,3 \pm 13,8$ & 0,281 \\
\hline $50-59$ anos & $202 \pm 50,2$ & $198,3 \pm 31,3$ & 0,660 & $44,1 \pm 13,7$ & $49,5 \pm 13,7$ & 0,062 \\
\hline$\geq 60$ anos & $185 \pm 29,3$ & $207,2 \pm 42,9$ & 0,008 & $42,1 \pm 11,2$ & $48,1 \pm 13,3$ & 0,028 \\
\hline Total & $180,3 \pm 43,5$ & $179,9 \pm 38,8$ & 0,905 & $43,7 \pm 15$ & $49,6 \pm 13,5$ & $<0,001$ \\
\hline $\mathrm{P}$ & $<0,001$ & $<0,001$ & 0,64 & 0,18 & & \\
\hline
\end{tabular}


A tabela IV demonstra os resultados obtidos nas duas pesquisas, no que se refere a outras variáveis analisadas. Como se observa, houve um decréscimo significativo nos níveis de pressão arterial, tanto sistólica quanto diastólica, e também no percentual de indivíduos em vida sedentária ou fumantes. Por outro lado, houve um aumento de $2 \mathrm{~kg}$ no peso médio dos entrevistados. Como seria de se esperar, pelas próprias características de seleção dos indivíduos entrevistados, os valores de idade e de sexo foram superponíveis.

A tabela V compara os resultados obtidos em 1991 e 1997, através de análises de regressão, entre as diversas

\begin{tabular}{|c|c|c|c|}
\hline \multicolumn{4}{|c|}{$\begin{array}{l}\text { Tabela III - Comparação entre o perfil do colesterol total (mg/dL) } \\
\text { aferido em 1991, em relação aos níveis de } 1997^{*}\end{array}$} \\
\hline & \multicolumn{2}{|c|}{ Ano } & \multirow[t]{2}{*}{$\mathrm{P}(95 \% \mathrm{IC}$ da diferença $)$} \\
\hline & 1991 & 1997 & \\
\hline Homens & $187,6 \pm 53,3$ & $190,5 \pm 42,5$ & $0,508(5,7$ a $-11,5)$ \\
\hline Mulheres & $196,8 \pm 44,2$ & $187,6 \pm 37,8$ & $0,008(2,4$ a 15,9$)$ \\
\hline Total & $192,5 \pm 48,9$ & $188,9 \pm 40$ & $0,192(9 \mathrm{a}-1,8)$ \\
\hline
\end{tabular}

\begin{tabular}{|c|c|c|c|}
\hline & \multicolumn{2}{|c|}{ Ano } & \multirow[t]{2}{*}{ P (95\% IC da diferença } \\
\hline & 1991 & 1997 & \\
\hline PAS $(\mathrm{mm} / \mathrm{Hg})$ & $134 \pm 23$ & $126,1 \pm 18,9$ & $<0,001(5,3$ a 10,4$)$ \\
\hline $\mathrm{PAD}(\mathrm{mm} / \mathrm{Hg})$ & $85 \pm 14$ & $81,6 \pm 12,3$ & $<0,001(1,8$ a 4,5$)$ \\
\hline Peso $(\mathrm{kg})$ & $67,7 \pm 13,7$ & $69,6 \pm 14,1$ & $0,024(-3,5$ a $-0,25)$ \\
\hline Altura $(\mathrm{cm})$ & $164 \pm 8,5$ & $164 \pm 10$ & $0,080(-2,1$ a 0,12$)$ \\
\hline Idade (anos) & $47,1 \pm 12,3$ & $46,9 \pm 12,8$ & $0,743(-1,24$ a 1,74$)$ \\
\hline Sexo masculino (\%) & 47,2 & 46,1 & $0,754(-7,1$ a 4,8$)$ \\
\hline Vida sedentária (\%) & 74,1 & 68,3 & $0,041(-11,1$ a $-0,4)$ \\
\hline Dieta $(\%)$ & 19,5 & 21 & $0,599(-3,3$ a 6,2$)$ \\
\hline Tabagismo & 31,5 & 24,7 & $0,015(-12,2$ a1,5) \\
\hline
\end{tabular}

\begin{tabular}{|c|c|c|c|}
\hline \multicolumn{4}{|c|}{$\begin{array}{l}\text { Tabela V - Análise de regressão das diversas variáveis } \\
\text { correlacionadas com níveis de colesterol nos anos de } 1991 \text { e 1997* }\end{array}$} \\
\hline \multirow[t]{2}{*}{ Ano da pesquisa } & 1991 & \multicolumn{2}{|c|}{1997} \\
\hline & $\mathrm{CT}$ & $\mathrm{CT}$ & HDL-c \\
\hline Idade & $\mathrm{p}<0,001$ & $\mathrm{p}<0,001$ & $\mathrm{p}=0,231$ \\
\hline PAS & $\mathrm{p}<0,001$ & $\mathrm{p}<0,001$ & $\mathrm{p}=0,278$ \\
\hline PAD & $\mathrm{p}<0,001$ & $\mathrm{p}<0,001$ & $\mathrm{p}=0,366$ \\
\hline IMC & não realizado & $\mathrm{p}=0,011$ & $\mathrm{p}=0,001$ \\
\hline Renda & $\mathrm{p}=0,119$ & $\mathrm{p}=0,348$ & $\mathrm{p}=0,733$ \\
\hline Escolaridade & $\mathrm{p}=0,946$ & $\mathrm{p}=0,774$ & $\mathrm{p}=0,562$ \\
\hline Sexo feminin & $\mathrm{p}=0,011$ & $\mathrm{p}=0,444$ & $\mathrm{p}<0,001$ \\
\hline Dieta (qualquer) & $\mathrm{p}=0,001$ & $\mathrm{p}<0,001$ & $\mathrm{p}=0,623$ \\
\hline Sedentarismo & $\mathrm{p}=0,116$ & $\mathrm{p}=0,014$ & $\mathrm{p}=0,502$ \\
\hline Tabagismo & $\mathrm{p}=0,147$ & $\mathrm{p}=0,096$ & $\mathrm{p}=0,731$ \\
\hline Estresse & $\mathrm{p}=0,818$ & $\mathrm{p}=0,756$ & $\mathrm{p}=0,177$ \\
\hline $\begin{array}{l}\text { *incluídos apena } \\
\text { 1991; não foi rea } \\
\text { PAD- pressão art }\end{array}$ & $\begin{array}{l}\text { íduos >29 ano } \\
\text { HDL-c em } 199 \\
\text { astólica; IMC- }\end{array}$ & $\begin{array}{l}\text { e mínima } \\
\text { - pressão } \\
\text { de massa }\end{array}$ & $\begin{array}{l}\text { esquisa de } \\
\text { al sistólica } \\
\text { ral. }\end{array}$ \\
\hline
\end{tabular}

variáveis estudadas e os níveis de colesterol total. No caso da pesquisa atual, foi realizada a mesma análise estatística para HDL-c, que não havia sido dosado na pesquisa anterior. Conforme observação, no colesterol total o comportamento das diversas variáveis foi similar nas duas pesquisas, com exceção de sexo feminino (correlação significativa em $1991 \mathrm{e}$ não significativa em 1997), e sedentarismo (correlação não significativa em 1991 e significativa em 1997). No que se refere aos níveis de HDL-c, as variáveis que se correlacionaram significativamente com os mesmos foram o índice de massa corporal (correlação negativa) e sexo feminino.

\section{Discussão}

\section{Análise dos dados obtidos na pesquisa de 1997.}

Em comparação com dados europeus do estudo PROCAM $^{21}$, os níveis médios de colesterol total obtidos em São José do Rio Preto foram menores, em ambos os sexos, para qualquer faixa etária analisada. No nosso material, obteve-se, no sexo masculino, valores que variaram entre $157 \mathrm{mg} / \mathrm{dL}$ e $202 \mathrm{mg} / \mathrm{dL}$ ( tab. II), enquanto no europeu esta variação foi entre $171 \mathrm{mg} / \mathrm{dLe} 230 \mathrm{mg} / \mathrm{dL}^{21}$. Nas mulheres, as variações foramentre $160 \mathrm{mg} / \mathrm{dLe} 207 \mathrm{mg} / \mathrm{dL}$ no nosso material (tab. II), e entre $182 \mathrm{mg} / \mathrm{dL}$ e $248 \mathrm{mg} / \mathrm{dL}$ no europe ${ }^{21}$. Por outro lado, a evolução do perfil do colesterol total, ao longo das décadas, foi similar em ambos os levantamentos: aumentos até a $6^{\text {a }}$ década para os homens, com posterior estabilização (ou até mesmo algum grau de decréscimo, no nosso material), e aumentos em todas as décadas analisadas, para as mulheres. Correlação positiva estatisticamente significativa $(p<0,001)$ foi demonstrada entre faixa etária e níveis de colesterol, no nosso material (tab. II). Os níveis médios de colesterol total na população analisada são semelhantes àqueles encontrados em países do Mediterrâneo, que variamentre $170 \mathrm{e} 213 \mathrm{mg} / \mathrm{dL}^{22}$.

Em nosso meio, duas publicações analisaram populações com idades médias similares à obtida no presente estudo (40,4 anos): Porto Alegre (40,7 anos) ${ }^{23}$ e São Paulo $(40,5 \text { anos })^{24}$. Os níveis de colesterol relatados foram discretamente superiores aos do presente estudo, $206 \mathrm{mg} / \mathrm{dL}$ e $189 \mathrm{mg} / \mathrm{dL}$ respectivamente, em relação aos $180 \mathrm{mg} / \mathrm{dL}$ aqui obtidos, o que talvez reflita a diferença entre os hábitos interioranos, em relação aos das capitais. Em publicação recente ${ }^{25}$, levando-se em conta indivíduos encaminhados para cinecoronariografia, encontrou-se colesterol total médio de $227 \mathrm{mg} / \mathrm{dL}$ nos indivíduos com coronariopatia obstrutiva, e de $204 \mathrm{mg} / \mathrm{dL}$ naqueles sem doença aparente.

Ao se analisar nossos dados em relação aos norteamericanos ${ }^{26}$, demonstra-se, da mesma forma, melhor perfil na população local. Por exemplo, nosso percentual de homens com colesterol total ideal, $\leq 200 \mathrm{mg} / \mathrm{dL}$, é de $71 \%$ (fig. 3), bem maior do que o obtido nos EUA, que variou entre $47 \%$ (americanos de ascendência mexicana) a 52\% (não-hispânicos). Para as mulheres, a mesma situação: $70 \%$ das brasileiras apresentavam níveis ideais de colesterol total, contra $51 \%$ em norte-americanas. 
Por outro lado, a prevalência de colesterol total $>240 \mathrm{mg} / \mathrm{dL}$ em homens norte-mericanos variou entre $15,7 \%$ e 17,8\% ${ }^{26}$, também maior que a obtida por nós, de $9 \%$ (fig. 3 ). Inversamente, esta prevalência em norte-americanas variou entre $17,5 \%$ e 20,2\%, sendo em S. J. Rio Preto de apenas 7\%.

Os níveis médios de HDL-colesterol obtidos no presente estudo se situam abaixo dos relatados no estudo PROCAM $^{21}$, para todas as faixas etárias analisadas. Em S. J. Rio Preto, tais níveis variaram entre $42,1 \mathrm{mg} / \mathrm{dL}$ e $43,7 \mathrm{mg} /$ $\mathrm{dL}$ para homens e entre $48,1 \mathrm{mg} / \mathrm{dL}$ e $51,3 \mathrm{mg} / \mathrm{dL}$ para mulheres (tab. II). No estudo europeu ${ }^{21}$, esta variação foi entre $45,4 \mathrm{mg} / \mathrm{dL}$ e $46,4 \mathrm{mg} / \mathrm{dL}$ no sexo masculino, e entre $56,9 \mathrm{mg} /$ $\mathrm{dLe} 58,4 \mathrm{mg} / \mathrm{dL}$ no feminino. Em ambos os estudos, ao contrário do que ocorreu com o colesterol total, os níveis de HDL-c foram similares nas diversas faixas etárias analisadas. Publicação nacional ${ }^{25}$ encontrou média de HDL-c de $39,1 \mathrm{mg} / \mathrm{dL}$ para indivíduos coronariopatas, e de $45,7 \mathrm{mg} / \mathrm{dL}$ para não-coronariopatas, este próximo aos $46,9 \mathrm{mg} / \mathrm{dL}$ do presente estudo. Além disso, em nosso material (fig. 4), $28,7 \%$ dos homens, e $12 \%$ das mulheres, apresentaram níveis de HDL-c não desejáveis, $<35 \mathrm{mg} / \mathrm{dL}$.

\section{Análise comparativa entre os dados obtidos em 1991 e 1997.}

A evolução temporal dos níveis de colesterol total apresenta grande variação na literatura. Algumas publicações demonstram aumento dos níveis ${ }^{27,28}$, outras demonstram diminuição ${ }^{29}$, enquanto outras ainda, similares aos nossos dados (tab. III), encontraram ausência de alterações significativas $^{30-32}$.

Além do mais, demonstram-se, conforme tabela IV, diminuições significativas nos percentuais de tabagistas e de fumantes, e nos níveis pressóricos, tanto sistólicos quanto diastólicos. Essas variáveis, como pode ser notado na tabela V, se correlacionam com níveis de colesterol.

Os dados da tabela III, no que se refere ao sexo, demonstram um aumento não significativo nos níveis de colesterol total nos homens e uma diminuição bastante significativa nas mulheres. Isto deve estar relacionado, ao menos em parte, ao fato de que as prevalências de mulheres tabagistas ( $36 \%$ vs $21 \%$, p $<0,001,95 \%$ IC $-22,2$ a $-7,1)$ e sedentárias $(79,5 \%$ vs $70,5 \%, \mathrm{p}=0,014,95 \% \mathrm{IC}-16 \mathrm{a}-2,1)$ diminuíram significativamente entre 1991 e 1997, ao contrário do que ocorreu nos homens, em que não foram encontradas diferenças significativas em relação às diversas prevalências analisadas. Além disso, tampouco foram detectadas diferenças significativas em relação ao peso, no sexo feminino $(64 \mathrm{~kg}$ vs $65 \mathrm{~kg}$, respectivamente nos dois levantamentos, $\mathrm{p}=\mathrm{NS}$ ), ao contrário do sexo masculino, em que houve um ganho significativo ( $72 \mathrm{~kg}$ vs $75 \mathrm{~kg}, \mathrm{p}=0,035,95 \%$ IC $0,2 \mathrm{a} 5,2$ ).

Em conclusão, os níveis de colesterol na população adulta de São José do Rio Preto são bastante aceitáveis e, no sexo feminino, houve um decréscimo significativo nos valores obtidos na pesquisa atual, em relação à realizada em 1991.

\section{Agradecimentos}

Aos Laboratórios Merck-Sharp \& Dhome pelo suporte financeiro, ao INCOR (Instituto do Coração-HCFMUSP), à FAMERP (Faculdade de Medicina de S.J. do Rio Preto), ao IMC (Instituto de Moléstias Cardiovasculares de S.J. do Rio Preto), à Prefeitura Municipal de S.J. do Rio Preto, à SMC (Sociedade de Medicina e Cirurgia de S.J. do Rio Preto), à FAPERP (Fundação de Amparo à Pesquisa e Extensão de S.J. do Rio Preto).

\section{Referências}

1. American Heart Association - International Cardiovascular Disease Statistics. Biostatistical Facts Sheets 1998: 1-7.

2. Meyer BJ, Meier B, Bonzel T et al - Interventional Cardiology in Europe 1993. Eur Heart J 1996; 17: 1318-28.

3. American Heart Association - Leading causes of death and age-adjusted death rate comparisons - 1995. Biostatistical Facts Sheets 1998: 1-5.

4. Ministério da Saúde - Coordenação de doenças cardiovasculares no Brasil. Sistema Único de Saúde. Brasília, 1993.

5. Lotufo PA, de Lolio CA - Tendência da mortalidade por doença isquêmica do coração no Estado de São Paulo. Arq Bras Cardiol 1993; 61: 149-53.

6. Lotufo PA, Benseñor IJ, Lolio CA - Tabagismo e mortalidade por doença isquêmica do coração. Estudo comparativo das capitais de regiões metropolitanas do Brasil, 1988. Arq Bras Cardiol 1995; 64: 7-9.

7. Grimm RH Jr - Treating hypertension and cardiovascular risk factors: are there trade-offs? Am Heart J 1990; 119: 729-32.

8. Stone PH, Sacks RM, Smith TW - Use of hypolipidemic strategies to halt atherosclerosis: evidence of alteration in clinical course. In: Grundy SM, Bearn AG, eds - The Role of Cholesterol in Atherosclerosis: New Therapeutic Opportunities. Philadelphia: Hanley \& Belfus, 1987: 83-103.

9. Sheperd J, Cobbe SM, Ford I et al, for the West of Scotland Coronary Prevention Study Group. Prevention of coronary heart disease with pravastatin in men with hypercholesterolemia. N Engl J Med 1995; 333 : 1301-7.

10. Scandinavian Simvastatin Survival Study Group - Randomised trial of cholesterol in 4444 patients with coronary heart disease: the Scandinavian Simvastatin Survival Study (4S). Lancet 1994; 344: 1383-9.

11. Sacks FM, Pfeffer MA, Move LA et al, for the Cholesterol and Recurrent Events Trial
Investigators. The effect of pravastatin on coronary events after myocardial infarction in patients with average cholesterol levels. N Engl J Med 1996; 335: 1001-9.

12. Giannini SD, Gois JM, Dereviacki BE et al - Prevenção primária em servidores do Hospital das Clínicas de São Paulo. Exposição dos achados em funcionários do Instituto do Coração. Rev Bras Med (Cardiologia) 1988; 3: 125-9.

13. Forti N, Santomauro AC, Jubelino FRS et al - Prevenção primária e doença arterial coronariana: identificação dos fatores de risco em servidores públicos da Municipalidade de São Paulo. Rev Bras Med 1990; 47: 343-54.

14. Pereira LSM, Souza OF, Carvalho PTR et al - Avaliação dos fatores de risco cardiovasculares em advogados do Rio de Janeiro. Rev SOCERJ 1992; 4: 120-4.

15. Bertolami MC, Faludi A, Latorre M et al - Perfil lipídico de empregados de indústria metalúrgica e sua relação com outros fatores de risco. Arq Bras Cardiol 1993; 63: 371-5.

16. Dioguardi GS, Pimenta J, Knoplich J et al - Fatores de risco para doenças cardiovasculares em médicos. Dados preliminares do projeto VIDAM da Associação Paulista de Medicina. Arq Bras Cardiol 1994; 62: 383-8.

17. Nicolau JC, Bechara DL, Nascimento SDG, Greco OT, Jacob JLB, Lorga AM Perfil do colesterol na cidade de São José do Rio Preto. Arq Bras Cardiol 1992; 59: 433-40.

18. Issa JS, Strunz C, Giannini SD, Forti N, Diament J - Precisão e exatidão das dosagens dos lípides sangüíneos em equipamento portátil (Cholestech-LDX). Arq Bras Cardiol 1996; 66: 339-42.

19. Wilkinson L Statistics. In: Wilkinson L, ed. Systat: The System for Statistics. Evanston, Systat Inc, 1990.

20. Glantz AS - Primer of Biostatistics. $3^{\text {rd }}$ ed. New York: McGraw-Hill, 1987.

21. Assman G, Schulte H - Results and conclusions of the Prospective Cardiovascular Münster (PROCAM) Study. In: Lipid Metabolism Disorders and 
Nicolau e col

Arq Bras Cardiol

Níveis de colesterol em São José do Rio Preto

volume 71, $\left(n^{\circ} 5\right), 1998$

Coronary Heart Disease, $2^{\text {nd }}$ edition. G. Assmann, ed. Munich: MMV Medizin Verlag GmbH München, 1993: 19-67.

22. Knuiman JT, West CE, Burema J - Serum total and high density lipoprotein cholesterol concentrations and body mass index in adult men from 13 countries. Am J Epidemiol 1982; 116: 631-42.

23. Duncan BB, Berger C, Silva MLS, Bassanesi SL, Achutti AC - Níveis séricos de colesterol em amostra representativa da população adulta de Porto Alegre. Arq Bras Cardiol 1988; 51: 385-90.

24. Forti N, Jubelino FRS, Santomauro AC, Novazzi JP, Salvetti XM, Biaggi R - Prevenção primária de doença arterial coronariana: I - níveis de colesterolemia em servidores da municipalidade de São Paulo. Atualização Cardiológica 1990; 2: res 121.

25. Forti N, Fukushima J, Giannini SD - Perfil lipídico de indivíduos submetidos à cinecoronariografia em diferentes regiões do Brasil. Arq Bras Cardiol 1997; 68: 333-42.

26. American Heart Association. Cholesterol. Biostatistics Fact Sheets 1998: 1-3.

27. Hoffmeister H, Mensink GB, Stolzenberg H - National trends in risk factors for cardiovascular disease in Germany. Prev Med 1994; 23: 197-205.
28. Pajak A, Williams OD, Broda G et al - Changes over time in blood lipids and their correlates in Polish rural and urban populations: the Poland-United States Collaborative Study in cardiopulmonary disease epidemiology. Ann Epidemiol 1997; 7: 115-24

29. Vartiainen E, Puska P, Jousilahti P, Korhonen HJ, Tuomilehto J, Nissinen A Twenty-year trends in coronary risk factors in north Karelia and in other areas of Finland. Int J Epidemiol 1994; 23: 495-504.

30. Wietlisbach V, Paccaud F, Rickenbach M, Gutzwiller F - Trends in cardiovascular risk factors (1984-1993) in a Swiss region: results of three population surveys. Prev Mec 1997; 26: 523-33.

31. Marques-Vidal P, Ruidavets JB, Ferrieres J, Bingham A, Cambou JP-Evolution des facteurs de risque cardiovasculaire chez l'homme en Haute-Garone, 1985-1987 et 1989-91. Resultats du Projet MONICA. Rev Epidemiol Sante Publique 1996; 44: 5-13.

32. Pekkanen J, Uutela A, Valkonen T, Vartiainen E, Tuomilehto J, Puska P Coronary risk factor levels: differences between educational groups in 1972-87 in eastern Finland. J Epidemiol Community Health 1995; 49: 144-9. 